\title{
REHAP Balance Tiles: a modular system supporting balance rehabilitation
}

\author{
Victor Donker \\ Department of Industrial Design \\ University of Technology Eindhoven \\ Eindhoven, The Netherlands \\ victor@victordonker.nl
}

\author{
Panos Markopoulos \\ Department of Industrial Design \\ University of Technology Eindhoven \\ Eindhoven, The Netherlands \\ p.markopoulos@tue.nl
}

\author{
Bert Bongers \\ Faculty of Design, Architecture and Building \\ University of Technology Sydney \\ Sydney, Australia \\ bertbon@xs4all.nl
}

\begin{abstract}
This paper describes the design, development, implementation and user evaluation of an interactive modular tile system, aimed to support balance rehabilitation of patients recovering from a stroke. The REHAP Balance Tiles system is an innovative tool, which has been developed in close collaboration with therapists and patients in stroke units of health rehabilitation institutes in Sydney, Australia and Eindhoven, the Netherlands. The system was designed to allow therapists to tailor exercises for each patient by changing the physical configuration of tiles. We report a user evaluation in a rehabilitation clinic, which lasted five weeks. Results indicate that the tiles can fulfill their envisioned purpose. They are received well by therapists, meeting requirements for ease of use, motivational feedback, modularity and flexibility.
\end{abstract}

Keywords - balance rehabiliation; motivation; evaluation; embodied interaction; field study and customization

\section{INTRODUCTION}

Stroke incidence is increasing and stroke survivors are very often left with considerable disabilities that require intensive rehabilitation. Technology can be a way to intensify training, reduce costs by allowing therapists to supervise several patients at the same time, and can potentially also improve training quality, making it less tedious and more intrinsically motivating. While early research on rehabilitation technologies concerned primarily rehabilitation robotics, there has been an increasing interest on tangible and embodied interaction, thanks to advances and the growing availability of sensing technologies. For example researchers have developed several such systems to support arm-hand training after stroke $[1,2,3,4]$ as well as balance training $[5,6,7]$.

Existing technological aids for balance training can be classified in two main categories: mechanical training aids and interactive training aids. Mechanical training aids are often more affordable and already widely used. While interactive products can still be relatively more expensive to purchase, they offer several advantages to patients regarding their interactivity such as informing patients about their activities by giving continuous and long-term feedback.

The REHAP Balance Tiles (see Fig. 1.) is a modular system that was designed for use during the rehabilitation process of stroke patients suffering from balance problems. The system consists of several pressure sensitive tiles; the largest one in the center is the 'main tile', to which several smaller 'sub tiles' can be connected. Because of this modular approach several physical layouts can be made in order to suit different needs of individual patients. The graphical user interface (GUI) directly reacts to the physical layout and shows the tiles as they are positioned. The pressure on the force sensors in the tiles is directly displayed, which informs patients of the pressure they put on a certain sensor with their body weight. The GUI serves as a communication tool for the patient and the therapist. In addition to providing feedback to the patient, it enables the therapists to explain the patients' own movement to the patient, as patients do not always feel what they actually do, or what their body posture is.

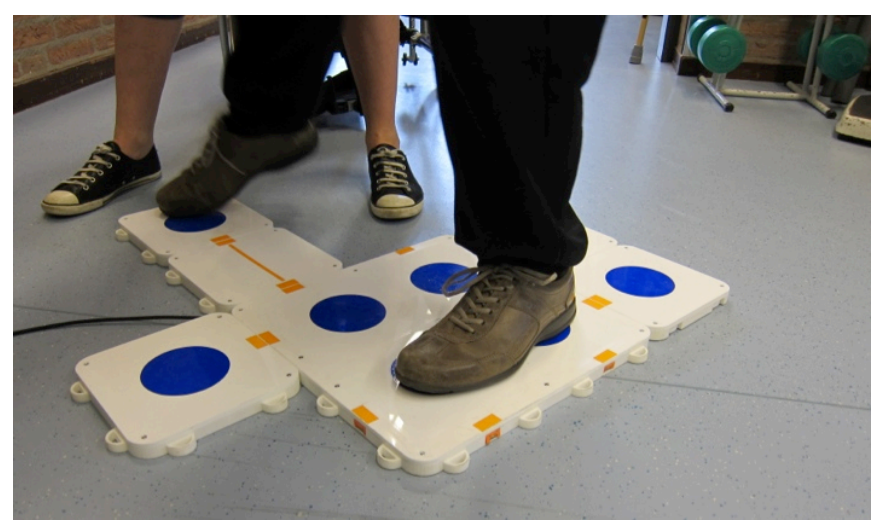

Fig. 1. REHAP Balance Tiles

The design concept was developed in an iterative and participatory design process involving medical researchers and therapists. Early on in this process a vision for personalisable products was formed. We chose a modular and interactive approach to fit the improvising way of working of therapists in the clinic to allow them to extend this in creating personalized exercises, addressing three essential patient needs from rehabilitation technologies customization, motivation, and independence [7]. By using sensors, actuators and interfaces in a modular way, we enable therapists to create a set of exercises per patient specifically, we expect that this will increase the motivation and possibility to train independently.

The tiles were developed over several iterations resulting in a prototype of high enough quality to withstand testing in the context of regular therapy. The prototype was field tested in several hospitals in Australia. A follow up evaluation was carried out in the Netherlands to evaluate the usability of the REHAP Balance Tiles. This test lasted five weeks; it confirmed the potential of the system though it identified several areas of improvement as detailed below.

\section{RELATED WORK}

In this section we present an overview of related research in three key research areas.

\section{A. Balance training}

Research in the field of balance training has often underlined the relevance of developing innovative strategies 
and products for the rehabilitation of stroke patients. Geurts et al. [8] state that is necessary to develop effective rehabilitation strategies for different types of stroke and show evidence of true physiological recovery of leg muscle function in postural control. Furthermore it is suggested that normal sensory integration is critical for balance recovery.

Karlsson and Frykberg [9] give an overview of variations and correlations between force plate measures to assess balance, but state that there is no indication of these plates being used as feedback instruments for patients.

Srivastava et al. [10] present a Force Platform with Visual Feedback (FPVF) that was used daily for four weeks by 45 people and show an improvement in balance and functional outcome in chronic stroke survivors. They state that training with FPVF significantly improves balance and functional outcomes even in the chronic phase after stroke.

Popovic et al. [11] derive a general model of the proposed four stability zones, which can be used to compute stability zones a priori for any subject and thus allows one to assess the subject's stability condition from the center of pressure measurements. Their proposed measure of stability compares the center of pressure measurements with the experimental stability zones: high preference, low preference, undesirable and unstable zones, and provides a quantitative measure of the subject's stability condition. This approach is suitable to be implemented in feedback control scheme to assess and maintain stability during standing.

Rugelj [12] shows effects of functional balance training and suggests that specifically targeted functional balance training is effective if functional activities are concerned.

\section{B. Visual Feedback}

Further research has proven the effect of visual feedback with several examples. Pao-Tsai Cheng et al. [13] state that visual feedback rhythmic weight-shift training significantly improves dynamic balance function for hemiplegic stroke patients.

Albiol-Pérez et al. [14] show the success of virtual reality in rehabilitation for patients with vestibular disorders. They state that visual feedback is very promising and accepted by patients and clinicians. The expectation is that visual feedback will increase people's motivation and independence.

\section{Modularity}

The final aspect to discuss is modularity, which is one of the main aims of the REHAP Balance Tiles. A vast range of systems in different application domains makes good use of modularity; we review some most related to the REHAP tiles.

Lund [15] shows a good example of a modular system called 'Playware'. Their system for playful physiotherapy is described and concludes that patients find the playful use of modular robotic tiles engaging and motivating in rehabilitation. Also, initial pilot test data suggest that some playful exercises on the tiles demand an average heart rate of $75 \%$ and $86 \%$ of the maximum heart rate, suggesting engagement with the system and the exercises. To compare, their visual feedback comes from the tiles themselves, while our approach of a separate screen at the patient's eye level responds to requirements from therapists, who indicated that to support balance training it was important that patients should look forward and up straight.

Bruce et al. [16] show a detailed example of a modular protocol. They state four key properties for the design and application of therapeutic interventions; partial decomposability, proper functioning, standardized interface, and information hiding.
The Z-Tiles by Richardson et al. [17] is a concept of modular tiles that can be used as control for music, dance or (virtual reality) gaming. Besides presenting tests and results for these purposes, they suggest their possible application for medicine and sports science, where a clinician could obtain a dynamic view of data in real-time. This information could be used to diagnose illnesses, correct problems with a gait, or improve athlete's performance.

\section{Conclusion}

From the related work we can see an emphasis on balance training, visual feedback and modularity for rehabilitation training equipment. An aspect that appears in several of the systems is visualizing the center of force, rather than visualizing separate pressure points, which is the approach taken with REHAP. Visual feedback has been proven to be beneficial for therapy. The modularity of such solutions has been argued to be important and promising to allow personalization to individual patient training needs, but is not yet common in systems for balance rehabilitation.

\section{DESIGN AND RESEARCH APPROACH}

An iterative 'research through design' approach has been followed, where quick design cycles are carried out in search for a suitable rehabilitation technology. The initial phases, carried out by student Rebecca Hall, were meant to identify the kind of training before the intervention. Later iterations were carried out to find out more about the feasibility of sensing, while later ones concerned the effectiveness of the feedback, the ease of use of the whole system and the fit to the training culture at the clinic. In total over 10 iterations were done, with frequent feedback moments in the stroke unit in an involved hospital. These iterations, involved to different extends the following stages:

\section{1) Observing}

Observations were conducted in the environment of training, with and without prototypes, to understand the problems from technological, social, medical, legal and financial

perspectives, to understand and identify how these aspects affects the needs of patients and therapists.

\section{2) Prototyping}

Simple prototypes or 'provotypes' [18] were produced rapidly, ranging from paper-prototypes to working artifacts with sensors and actuators.

\section{3) Testing}

Prototypes are tested and evaluated with the users in context. These evaluations varied from simple informal discussions to structured questionnaires. The integration of testing different variations of technology helped to see the responses of professionals.

\section{4) Analyzing}

Test results and feedback are analyzed to guide iterative improvements of the design concept.

\section{5) Redesign}

From the results of the test analysis the prototypes were redesigned in each iteration, resulting in smaller or bigger improvements, feeding into the next iteration.

This iterative development was done in the Stroke Unit of in aHospital in Bankstown, a suburb of Sydney, Australia. The subsequent evaluation described later in this paper was conducted at the Libra Revalidatiecentrum Blixembosch, Eindhoven in the Netherlands. This evaluation took a different more summative approach, testing the robustness of the system, as well as its effectiveness and usability in context. 

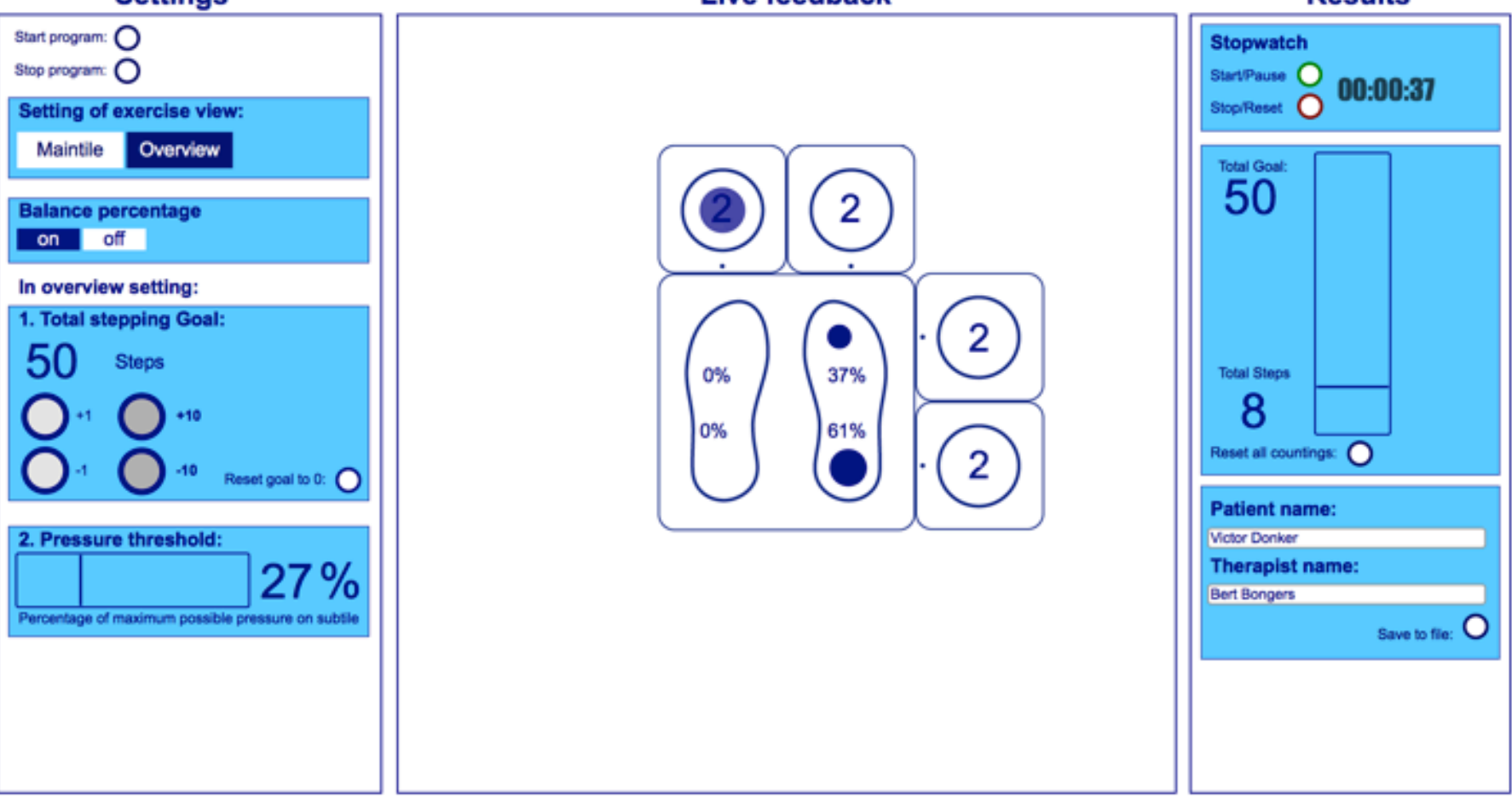

Fig. 2. Graphical User Interface.

\section{REHAP BALANCE TILES}

Following the process outlined above we developed a modular set of balancing and stepping task tiles with a connected display. The work was partly inspired by the exercise mat [19], a design adapted from the popular Dance Dance Revolution game. Using this mat, with embedded pressure sensitive switches, elderly or less mobile people can practice dance and step exercises at home. Digital games for physical therapy have been used successfully in other projects [20].

The main structure of the REHAP tiles is 3D-printed, which offered several possibilities to quickly adapt prototypes to test in practice during the design. Layers of acrylic material achieve torsional strength, which are laser cut to size. Between de acrylic layers is a foam layer that is used to increase the sensibility surface of the tile. The tiles are in total $20 \mathrm{~mm}$ high, which is much lower than balance hardware used for entertainment such as the Wii Balance Board, making it easier and more secure for patients to step and stand on. The main tile is $400 \times 400 \mathrm{~mm}$, and measures the pressure of 4 points using Force Sensitive Resistors (FSRs), the back and front of the left and right feet. The exercise surface of the main tile can be extended with smaller 'sub tiles'. The connection is made with spring-loaded magnetic connections from LittleBits [21]. These sub tiles with one single force sensor are 200x200mm, and therefore in total 8 sub tiles can be placed around the main tile. The sub tiles allow therapists to create stepping exercises, which are crucial for weight shifting, an important exercise to support the ability to functionally walk again. To allow longer steps there are also connection tiles; these tiles do not have a pressure sensor, but pass on the signal of a connected sub tile.

\section{A. Graphical User Interface}

The Graphical User Interface (GUI) is built to control the settings of the exercise and overview, the real-time feedback and a results section. A screenshot of the GUI can be seen in Fig. 2. The interface is an information and communication system for both the patient and the therapist. The graphical illustration of growing dots based on increasing pressure is intended to be simple, yet very informative representation during balance and stepping exercises for stroke patients.

The on-screen interface responds directly to the layout of the physical tiles on the floor. The sub tiles are immediately displayed on the screen when connected to the main tile. Furthermore the amount of stepping repetitions on those single tiles is counted and shown. In the settings part, the user can also choose the threshold and activate percentages of single pressure points, relatively to the total pressure. Next to this the user can set the goal of amount of steps to be taken in total. The right part of the GUI shows a stopwatch and the progression of the total amount of steps toward the stepping goal. This seemed to be very valuable for therapists, as they like to have an overview of how the patient increases the average amount of steps per minute.

For logging purposes the interface also allows to fill in the patient and therapist names, and a possibility to write notes of the exercise. When pressing the save button, a log file will be automatically produced, with information about that certain exercise, creating a personal electronic health record. These files can be opened in a spread-sheet program such as Microsoft Excel and can be used to obtain graphical overviews of the process. Keeping an overview of the progression of a patient over a long time will help the guidance and will give a better view the achievements on long term.

\section{PRELIMINARY EVALUATION}

A preliminary (non clinical) evaluation with therapists and patients at the Libra Revalidatiecentrum Blixembosch was aimed to evaluate the usability of the system, and its potential acceptance by patients. It was not meant to test the 
effectiveness of training, for which a clinical trial will be necessary.

To evaluate the usability, the tiles have been placed in a rehabilitation center for five weeks. Therapists used the tiles voluntarily, in order to fit it in their regular therapy they use to support patients with balance problems. Planning certain moments in their schedule would not work out, as therapists need to spend all their time on training their patients. Furthermore, most patients want to focus on their rehabilitation and could feel interaction as an invasion of their privacy.

According to feedback, the simple displayed information in front of the patient, showing a continuous signal, of the pressure points has more value than only showing the balance right/left. This information is also a big advantage for the therapist, as it can be used as a communication and explanation tool, as shown in Fig. 3.

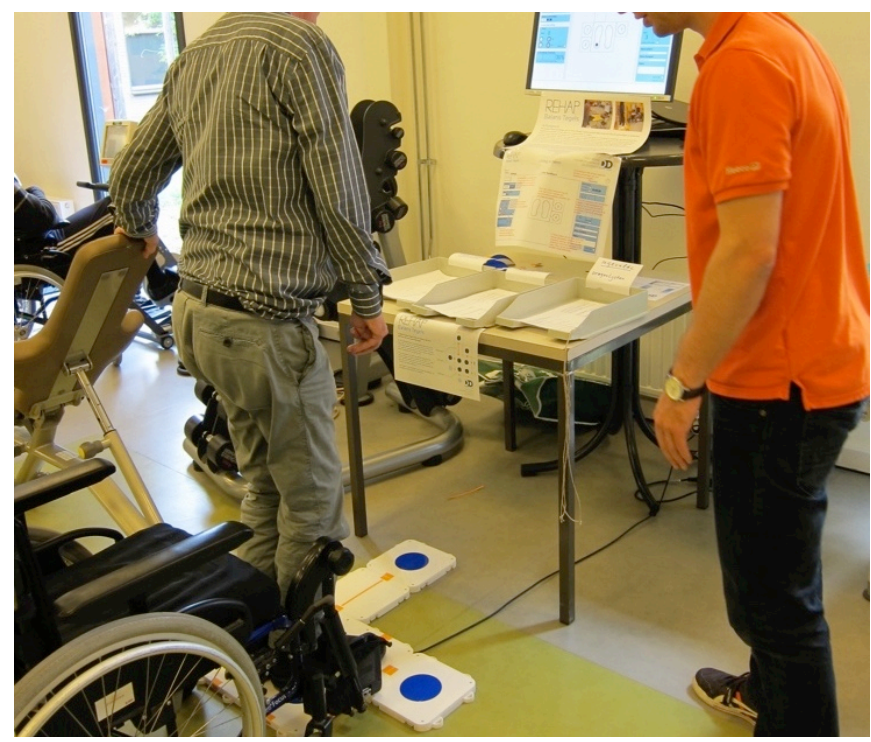

Fig. 3. Patient exercising with therapist assistance

One other advantage of having a modular system was noticeable when therapists asked if the tile could be used in a tilt table bed exercise, in which patients have to push themselves up in a bed by pressing against the footboard of the bed, as seen in Fig. 4. Getting fine-grain feedback on the amount of pressure on the back and front of both feet helps patients to train the stability and strength in their legs.

For example, one patient was not able to press up at all although he got very motivating feedback from the therapist. He was not aware and could not believe he had the ability and power to do so. When using the main tile and the GUI the patient saw a little dot, which was the visual feedback of his own movement, helping him believe in himself and motivating him to press even harder. Just a simple growing dot was of great motivational value for this patient. Observations and notes were taken during exercises and afterwards in short conversations with therapists. To collect qualitative and quantitative data regarding their experiences and suggestions, questionnaires were placed close to them during the intervention. The observations, conversations and answers of the questionnaires are discussed in the Results section.

\section{A. Method}

The designer acted as a participant observer, shadowing therapists as they trained different patients and conducting contextual interviews in the general approach sketched out by Beyer and Holtzblatt [22] and Harris [23]. For the final evaluation, questionnaires were developed iteratively in consultation with the clinic, so that they would be acceptable to therapists and to the ethics board of the clinic. The final questionnaire aimed primarily to assess the motivation of the patients to train with the use of the REHAP Balance Tiles. At the end of training sessions the designer asked whether therapists and patients would mind to complete the questionnaires.

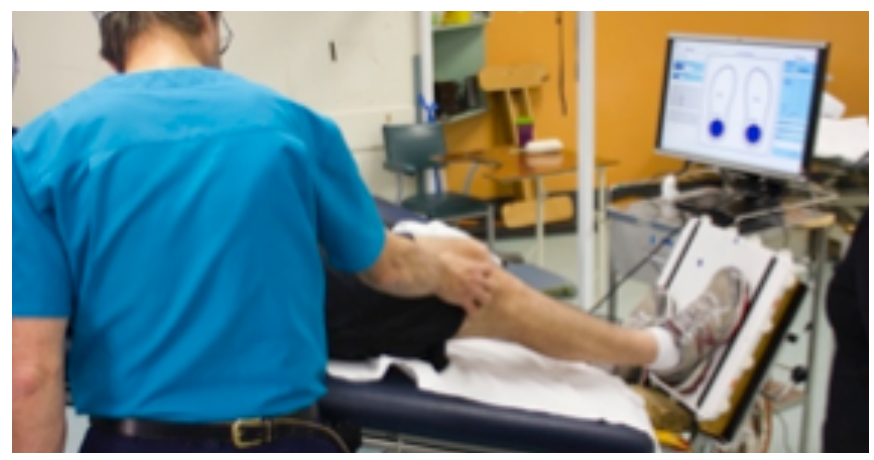

Fig. 4. Main tile being used in tilt table bed exercise

\section{B. Participants}

\section{1) Therapists}

Participating therapists had many different levels of expertise and experience. Furthermore they were working in different rooms, offering varying levels of guidance to patients. In total 10 therapists used the tiles on a voluntarily base during its 5week long deployment in the fitness and exercise rooms. 6 therapists completed the questionnaire. Several others consulted it and gave some verbal feedback, but did not fill in the questionnaires.

\section{2) Patients}

There was a lot of variation with regards to the pathologies of patients engaging in balance training during our test. Balance problems can be a symptom in many diseases, varying from thrombosis or stroke to Parkinson. All patients were between 31 and 70 years old. The amount of patients participated is hard to estimate, as some therapists used it for different patients. In total, only 5 patient questionnaires were completed as completing it was left totally to their discretion.

\section{Apparatus and Materials}

The REHAP Tiles and the screen were placed on a higher standing table in the fitness and exercise rooms with the idea that it should fit in the regular activities of practitioners. In both rooms the tiles were placed in the current setting, as they should be used in daily use (see Fig. 5 and 6). This was done with the idea that the Tiles should be used in a trusted environment rather than an unknown and clinical lab setting.

For data recording, observations and pictures were taken. Furthermore notes were taken during discussions with different therapists and patients. These interviews were informal and semi-structured aiming for qualitative information about the opinions of the users. Next to this, the questionnaires were presented in boxes on a table, so participants could provide more structured feedback by writing down their ideas on paper.

\section{Procedure}

The entire therapist team was invited to a presentation, where they were introduced to the system. The team also 
received a few emails during the weeks with a reminder about the possibility to use REHAP voluntarily. These emails included short description and pictures. Some papers with explanations of the project and REHAP were located next to the setup. In the first weeks, the tiles were in the fitness room, as seen in Fig. 5. This fitness room is a rather traditional fitness environment where patients can (individually) do exercises on treadmills, bikes, and other exercise equipment.

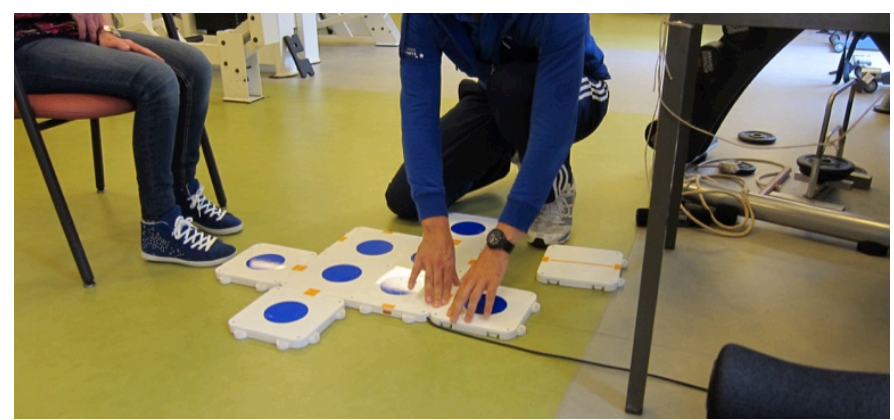

Fig. 5. REHAP Balance Tiles in fitness room context

The second place for REHAP training was an exercise room, where it was used for an additional two weeks, (see Fig. 6). The major difference between the fitness and exercise room is that patients get more personal guidance in the latter. Patients went through a guided session by one or two therapists of half an hour in which a tailored set of exercises were done with the patient in a more practical manner. People are for example simply walking the stairs or standing up.

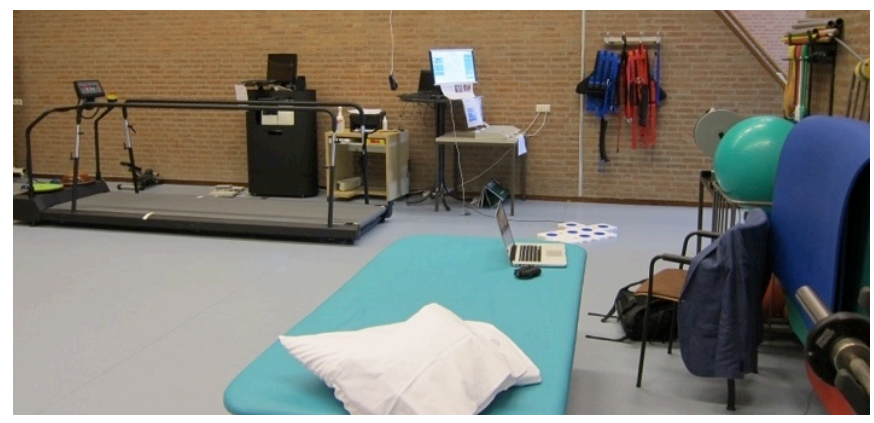

Fig. 6. REHAP Balance Tiles in exercise room context

\section{E. Measures}

The questionnaire consisted of several open questions, including 7-point rating scale questions and demographic questions. The questions were mainly on the topics of applicability, ease of use, helpfulness of the visual feedback, the motivation for patients, the possible independence and the successfulness for the treatment. An example question, translated from Dutch:

Does the pressure visualization on the screen help the patient to train?

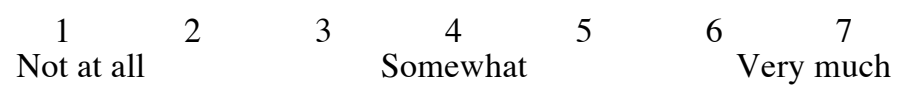

Therapists were asked to fill in the questionnaire, if possible together with their patient, after using REHAP. This was done because patients were unable to fill in a questionnaire themselves. In some situation the therapists wrote down the words of the patients. There was enough space for suggestions per question and overall at the end, to be written down without obligation.

\section{RESULTS}

The results of the questionnaires are shown below, separated in the therapists' and patients' answers.

\section{A. Therapists}

First we look at the results coming from the therapist (Table I). Six rating scale questions were asked, all having 7 options. Therapists rated the applicability of REHAP in their regular approach above average. The ease of use of the tiles and the interface was found easy. The usefulness of the GUI was also found influencing in a positive way. The highest score is given for the opinion that REHAP motivates the patients. On the question if they would expect that patients could train independently the answer is in the middle of the scale. The success for reducing symptoms was found above neutral.

TABLE I. THERAPIST QUESTIONNAIRE REULTS

\begin{tabular}{|l|c|}
\hline \multicolumn{1}{|c|}{ Question topic } & $\overline{\mathbf{X}}(\boldsymbol{\sigma})$ \\
\hline Applicability & $4,67(0,83)$ \\
\hline Ease of use & $5,67(1,34)$ \\
\hline Helpfulness of visual feedback & $5,67(1,22)$ \\
\hline Motivational for patient & $5,83(1,1)$ \\
\hline Independence & $4(1,52)$ \\
\hline Successfulness for treatment & $4,17(0,89)$ \\
\hline
\end{tabular}

Open questions were asked as well; a summary of responses is shown in Table II. Half of the therapists answered that REHAP fulfilled their expectations. One of the answers included the comment that more time was needed to try it. The other two negative answers mention the limit of use, because of the tiles and foot sizes. Positive answers commented on the ease of use, the advantages of the graphical feedback and even mentioned a patient improving in one session.

Five out of the six therapists said that they would use REHAP during their treatments. The most mentioned comment is that it the invention gives pleasant, positive, and motivating visual feedback for patients. Critical notes concerned the size of the tiles compared to the feet and the amount of tiles, which could be improved. Looking to the target groups, stroke patients would benefit most from REHAP according to the therapist feedback.

TABLE II. PATIENT QUESTIONNAIRE RESUlTS

\begin{tabular}{|l|c|}
\hline \multicolumn{1}{|c|}{ Question topic } & Fraction yes \\
\hline Fulfilled expectation & 0,50 \\
\hline Willing to use REHAP & 0,83 \\
\hline
\end{tabular}

\section{B. Patients}

Furthermore, we have data from the questionnaires filled in by five patients (see Table III). Firstly the yes/no questions 
show that all patients have used REHAP under guidance. $80 \%$ of the subjects mentioned that they got a better feeling of their body movement because they got visual feedback displayed and $60 \%$ expects that training with REHAP would help them to decrease their balance problems.

TABLE III. RESULTS LIKERT QUESTIONS PATIENT

\begin{tabular}{|l|c|}
\hline \multicolumn{1}{|c|}{ Question topic } & Fraction \\
\hline Trained independently & 0,00 \\
\hline $\begin{array}{l}\text { Better feeling because of visual } \\
\text { feedback }\end{array}$ & 0,80 \\
\hline $\begin{array}{l}\text { Expectation success, decrease of } \\
\text { balance problems }\end{array}$ & 0,60 \\
\hline
\end{tabular}

The results of the two seven point rating scale are summarized in Table 4. The first question was about motivation and the second about how gladly they would like to use REHAP. Both show a positive result. Stimulation is high for patient exercising with REHAP and the result is satisfying enough to say they would like to use REHAP in future sessions.

TABLE IV. TABLE 4. Results LiKeRT QUESTIONS PATIENT

\begin{tabular}{|l|c|}
\hline \multicolumn{1}{|c|}{ Question topic } & $\overline{\mathbf{X}}(\boldsymbol{\sigma})$ \\
\hline Stimulation & $5,8(1,79)$ \\
\hline Interest to use REHAP & $5,4(2,07)$ \\
\hline
\end{tabular}

With regards to the question "Do you feel better how you change your weight because of the information on the screen?", an overall answer would be: "Yes the visual feedback, the blue circles, help me to see my position, because I don't feel that myself. The circles are a clear visual representation, but not always easy to see because I also want to see how I need to position my feet".

Looking to the aspects that patients found useful and pleasant, overall the answer is that the balance is improved because of the visual feedback that gives information and confidence about balance. One patient even mentioned that she already improved in the second round of using it.

\section{DISCUSSION}

The completed questionnaires, extensive observations and informal conversations gave a qualitative overview and reinforce the design rationale for the REHAP Balance Tiles. Overall opinions expressed were that the concept is promising; that it offers various options for training and that multiple kinds of pathologies can benefit form using the REHAP tiles. The visual feedback was found particularly suited for training with stroke patients.

Being able to make custom exercises is of importance for most therapists and more additional tiles were requested by several of them. This resonates with [4] who focused on exercise customization using a graphical user interface and allowing therapists to tag physical objects. Here customization is based on varying the placement and topology of tile compositions as this is supported by the modularity of the REHAP system. Having more tiles would allow therapists to make more complex and challenging exercises and have patients eventually make bigger or more steps, allowing training towards the walking movement. The most necessary improvement concerns the sensitivity of pressure sensors in the tiles. Currently patients need to stand at the center of the tiles, with their feet nicely centered on the blue circles. Most therapists said that a larger pressure sensitive surface would be better for most patients.

Looking at the evaluation itself a few aspects could be improved in future tests as well. First, rather than the short questionnaire used more formal psychometric instruments could have used to measure patient motivation [24], the credibility of the system as a rehabilitation technology [25], and acceptance [26]. Unfortunately, this would result in a much more extensive questionnaire which was not acceptable to the clinic for practical and ethics reasons. A difference in use between the fitness room and the exercise room was noticed in the way that therapists used the exercise room for more practical exercise. This room has the space for people to walk around, throw balls or walk the stairs. A more flexible use of the system will be made possible with a wireless connection to a display, which would be easily moved around by therapists and patients alike. Currently the system is used with a connection to such a display.

In order to respect patient's privacy, they were not approached to test the tiles during their training and therefore it is not clear how many patients actually benefited from using them or not. When questioned, some patients said that they did not benefit from using the system. By contrast, others were overly enthusiastic, calling it a 'super device' and that they would like to use it in the further stages of their rehabilitation. This suggests that the more structured clinical trial would help getting a better understanding of patient attitudes and the effectiveness of using the tiles in training. As confirmed by therapists, some patients improved their body movement and stability within a few minutes of using the system. As an example, one of the patients (see Fig. 7) had to do the stepping exercises on four tiles clockwise and had to repeat this circle five times. The first steps she was very unsure and guidance of two therapists was needed. During the last round he carried out exercises very smoothly and independently. Of course, this can be largely attributed to learning the specific motion rather than improving balance skills, but it remains an indication of the effectiveness of training with this device.

Patients appeared to be motivated by the interaction with the device, which helped them improve the movement in training. However, this positive result is tentative and is studied further during a larger and longer-term study carried out by a team of researchers in Australia, funded by the NHMRC (National Health and Medical Research Council). This research project, led by Prof. Cathie Sherrington, carries out a controlled study with 400 participants, comparing interactive tools with traditional interventions. The Tiles are one of the interactive tools tested, and the first pilot study (with 45 participants) presented the benefits of interactive rehabilitation. 


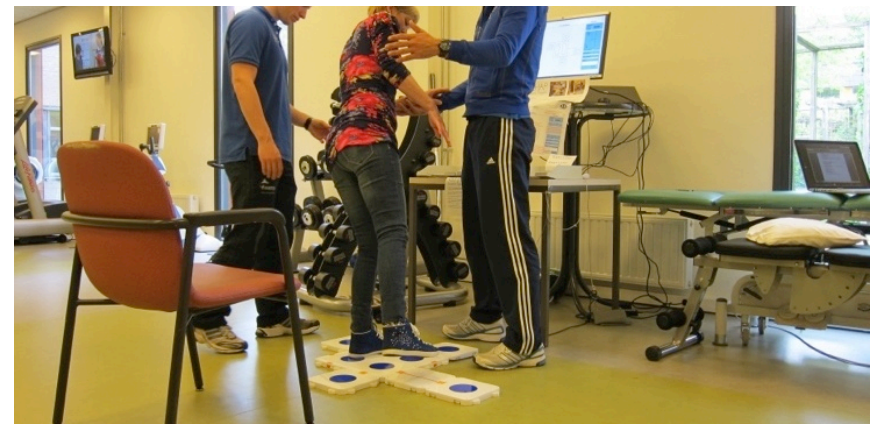

Fig. 7. Patient stepping

\section{VIII.CONCLUSION AND FUTURE WORK}

The contribution of this research is the introduction of a novel innovation, co-developed and evaluated in the context of practical rehabilitation. It is promising that the tiles have been used for a longer time in different institutes and that patients show improvement even during one single exercise. The evaluation shows that therapists show big interest towards customizing exercises by connecting different tiles in specific configurations for each patient. Furthermore, the visual communication has been evaluated as effective and motivating for patients.

Based on experiences from the development process and our results we found that it is essential to go into the context early in the process to find the strengths and needs of the user. By observing the way of working, proposing ideas by bringing physical and 'quickly-made ideas' it is easier to test, analyze and discuss to find several key factors to design for. Looking to the fact the tiles been proven to be very valuable for patients and therapists in practical context, especially the aspect of personalizing products and systems by using a modular approach is an essential value.

The innovative part of this solution is the combination of essential visual feedback and the possibility to create customized exercises for individual patients because of the modularity. We argue that the REHAP Balance Tiles is a promising tool, because of their adaptability and modularity but still require some improvements in order to reach a wider acceptance by its users. These improvements are presented below.

\section{A. Sensitivity}

The tiles were designed to be very sensitive, and with a threshold that can be set in the GIU enabling picking up of even the slightest touch of the foot of the patient if needed. An important aspect is the different sizes and position of the feet of patients; therefore bigger sensitive areas per feet are needed. We are currently investigating this through the development of a testing rig that we used to carry out repeated measurements across the surface of each tile, mapping out the sensitivity, and explored improvements through the composition and distribution of the foam material, which disperses the force applied.

\section{B. More tiles}

A simple improvement would also be to give more sub tiles and a variety of connection tiles, allowing for more and longer steps, which is required for advanced exercising. The purpose of redesigning of the 3D model of the tiles in a parametric model is to be able to generate different sizes, to cover longer distances if needed.

\section{Sound}

We have been working on designing appropriate sonic feedback on the balancing tasks and progress over time. Using sound as a feedback modality links well to the requirement that the patients don't have to look at the tiles during their exercises. The sounds we are developing are dynamic (nonrepetitive) and natural, rather than annoying beeps, and aim to blend in the soundscape of the ward.

\section{Overall}

Future research could be done after the previous mentioned improvements are made. When having a new prototype (including aspects like sound feedback, a bigger sensitive surface and additional tiles) a group of therapists and patients should be recruited who are willing to work with the Tiles on a structural base over a longer time to improve for future versions. Currently development in Australia is done to create a smaller tile that is connected to an iPhone as, See Figure 8.

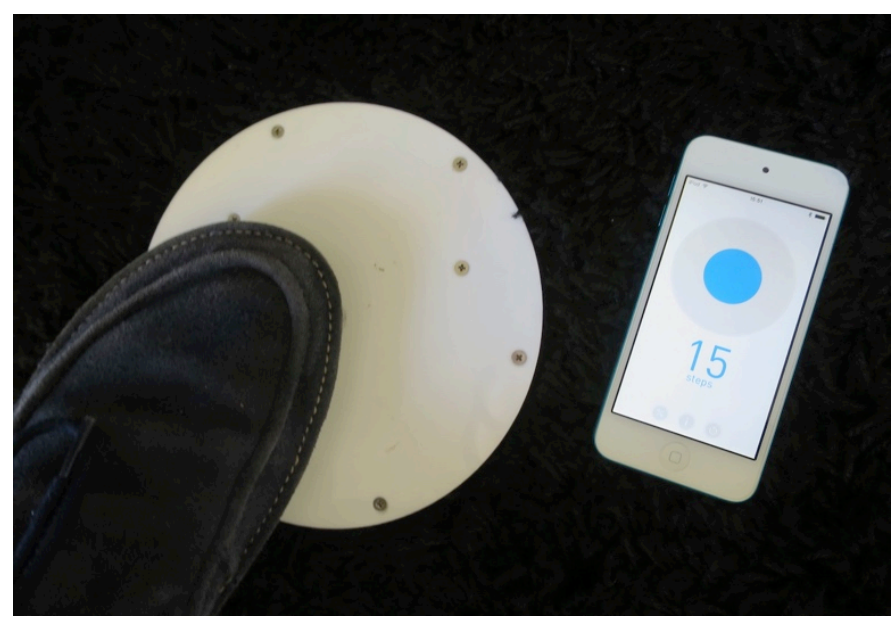

Fig. 8. Patient stepping on App version

By using smaller elements, different possibilities like home training can be created. With these building blocks, patients could be easier train as home which could enlarge the effect of training, as people can do this on a daily base. The development of these options is still going. Overall the focus will stay on creating modular solutions to enhance the motivation for individual patients in order to rehabilitate better from balance difficulties due to a stroke.

\section{ACKNOWLEDGMENTS}

We would like to thank among others especially the therapists and patients at Libra Revalidatiecentrum Blixembosch (Eindhoven), User Centered Engineering Research Group (Eindhoven), CCDP (UTS, Sydney), George Institute for Global Health (Sydney) and the Bankstown-Lidcombe Hospital (Bankstown, Sydney).

\section{REFERENCES}

[1] Pickrell, M. and Bongers, A. J. Re-Ability Sleeve - A Modular Wearable Rehabilitation Interface System. Workshop paper SmartHealth, OzCHI 2011.

[2] Jacobs, A., Timmermans A., Michielsen, M., Platse, Vander, M., Markopoulos, CONTRAST: Gamification of Arm-Hand Training for Stroke Survivors. CHI 2013.

[3] Delbressine F., Timmermans A., Luuk Beursgens L., Jong, de M., Dam, van A., Verweij D., Janssen M., Markopoulos P., Motivating arm-hand use for stroke patients by serious games. IEEE EMBS. 
[4] Tetteroo, D., Timmermans, A. A., Seelen, H. A. and Markopoulos, P. (2014). TagTrainer: supporting exercise variability and tailoring in technology supported upper limb training. Journal of neuroengineering and rehabilitation, 11(1), 140 .

[5] Sherrington, C., Whitney, J.C., Lord, S.R., Herbert, R,D., Cumming. R.G., and Close. J.C., Effective Exercise for the Prevention of Falls: A Systematic Review and Meta-Analysis. The American Geriatrics Society, 56 (12), (2008).

[6] Bongers, A. J., Smith S. T., Donker, V., Pickrell. M. and Hall, R. Interactive Infrastructures - Physical Rehabilitation Modules for Pervasive Healthcare Technology. In: A. Holzinger, M. Ziefle \& C. Röcker (Eds.) Pervasive Health - State of the Art and Beyond. Springer, 2014.

[7] Bongers, A. J., Smith S. T., Pickrell. M., Hall, R. and Donker, V. Interactivated Physical Rehabilitation Modules. Proceedings of the International ACM Creativity and Cognition Conference (2013).

[8] Geurts A. C. H., Haarta, M. de, Nesa I. J. W.van, Duysensa J. A review of standing balance recovery from stroke. Gait \& Posture 22, 2005, Elsevier

[9] Karlsson A., Frykberg G. Correlations between force plate measures for assessment of balance. Clinical Biomech, 2000, pp. 365-9

[10] Srivastava A., Taly A. B., Gupta A., Kumar S., Murali T. Post-stroke balance training: Role of force platform with visual feedback technique. Journal of the Neurological Sciences, Elsevier., 2009, pp. 89-93

[11] Popovic tM.R., Pappas I.P.I., Nakazawa K., Keller T., Morari M., Dietz V. Stability criterion for controlling standing in able-bodied subjects. Journal of Biomechanics 33.,Nov 2000, pp. 1359-68

[12] Rugelj D. The effect of functional balance training in frail nursing home residents. Archives of Gerontology and Geriatrics 50., Mar-Apr 2010, pp. 192-7

[13] Pao-Tsai Cheng, Chin-Man Wang, Chia-Ying Chung and Chia-Ling Chen. Effects of visual feedback rhythmic weight-shift training on hemiplegic stroke patients. Clinical Rehabilitation, 2004, pp. 747-53

[14] Albiol-Pérez S., Gil-Gómez J.A., Alcañiz M., Llorens R., Colomer C. Use of the Wii balance board system in Vestibular Rehabilitation. Interaccion '11 Proceedings of the 13th International Conference on Interacción Persona-Ordenador, Article No. 11 (ACM New York), 2012
[15] Lund H. H. Modular robotics for playful physiotherapy. 2009 IEEE 11th International Conference on Rehabilitation Robotics

[16] Bruce F. Chorpita, Eric L. Daleiden, John R. Weisz. Modularity in the design and application of therapeutic interventions. Applied and Preventive Psychology 11 (2005) 141-156

[17] Richardson, B., K. Leydon, K., Fernström, M. and Paradiso, J. (2004) ZTiles: Building Blocks for Modular, Pressure-Sensing Floorspaces. In Proc CHI 2004, ACM press.

[18] Boer, L., Donovan, J., Provotypes for Participatory Innovation. DIS 2012, June 11-15 2012, Newcastle, UK.

[19] Smith S. T., Sherrington C., Schoene D., Studenski S. and Lord S. R. A novel Dance Dance Revolution system for in-home training of stepping ability in older adults. British Journal of Sports Medicine, 45(5), 2011, 441-445.

[20] Geurts, L., Abeele, V. van den, Husson, J., Windey, F. Overveldt, M. van, Annema, J. H. and Desmet, S., Digital Games for Physical Therapy: Fulfilling the Need for Calibration and Adaptation. Proceedings of the TEI'11 conference, 2011, 117 - 124.

[21] Bdeir, A. and Ullrich, T. Electronics as Material: littleBits. Proceedings of the TEI '11, 2011, 341-344.

[22] Beyer H., Holtzblatt K. Contextual Design, Defining Customer-Centered Systems. Morgan Kaufmann, 1998, Chapter 9, Creating One View of the Customer

[23] Harris P. Open Guides to Psychology: Designing and reporting experiments. Published by Open University Press, 2002

[24] Pelletier, L.G., Tuson, K.M. Haddad. M.K., Client Motivation for Therapy Scale: A Measure of Intrinsic Motivation, Extrinsic Motivation, and Amotivation for Therapy. Journal of Personality Assessment 68 , Apr 1997, pp. 414-35

[25] Devilly, G.J., Borkovec, T.D., Psychometric properties of the credibility/expectancy questionnaire. Journal of Behavior Therapy and Experimental Psychiatry Vol. 31., Jun 2000, pp. 73-86

[26] Venkatesh, V., Morris, M.G., Davis, G.B., Davis, F.D., User Acceptance of Information Technology: Toward a Unified View. MIS Quarterly, Vol. 27, No. 3, Sep 2003, pp. 425-478 